\title{
Reduced Complexity QoS aware Resource Allocation Technique for MISO-OFDMA Systems
}

\author{
Mahmoud M. Selim*, Said Elnoubi*, Hossam Shalaby*, Osamu Muta ${ }^{\dagger}$, Hiroshi Furukawa ${ }^{\ddagger}$ \\ *Egypt-Japan University of Science and Technology (E-JUST), New Borg Al-Arab, Alexandria, Egypt \\ Email: mahmoud.sleem@ejust.edu.eg, saidelnoubi@yahoo.com, shalaby@ieee.org \\ ${ }^{\dagger}$ Center for Japan-Egypt Cooperation in Science and Technology, Kyushu University, Fukuoka-shi, Fukuoka, Japan \\ Email: muta@ait.kyushu-u.ac.jp \\ ${ }^{\ddagger}$ Graduate School of Information Science and Electrical Engineering, Kyushu University \\ Email: furuhiro@ait.kyushu-u.ac.jp
}

\begin{abstract}
In this paper, we propose a reduced complexity resource allocation technique for the downlink of Multiple-Input Single-Output Orthogonal Frequency Division Multiple-Access (MISO-OFDMA) system. The proposed algorithm efficiently allocates frequency resources among users per subcarrier such that a group of users are simultaneously assigned on the same subcarrier with minimum amount of interference through zero forcing beamforming (ZFBF). The proposed algorithm considers quality of service $(\mathrm{QoS})$ in terms of minimum user rate for each user and preserves fairness among users. QoS plays a key role in fulfillment of users demand of high bandwidth data services in modern wireless systems. Simulation results reveals that the proposed algorithm outperforms other reference algorithms in terms of sum rate, minimum user rate, $Q 0 S$ and preserves a very good fairness performance. Complexity of proposed algorithm is measured by execution time needed and compared to reference algorithms. Simulation results show a further reduction in complexity.
\end{abstract}

\section{INTRODUCTION}

Orthogonal Frequency Division Multiple-Access (OFDMA) is a premier technology in most of modern wireless standards such as IEEE 802.16e (mobile WiMAX) and Long Term Evolution-Advanced (LTE-Advanced). OFDMA is capable of fighting multipath fading and supporting high data rate services by dividing wideband channel into orthogonal flat fading narrowband subchannels [1].

The combination of Multi-Input Multi-Output (MIMO) technology with OFDMA (i.e. MIMO-OFDMA) increases system capacity [2] at the expense of higher system complexity due to the hardness of allocating system resources efficiently among users. The main goal of any efficient resource allocation technique is either to maximize system capacity or minimize total transmit power [3].

Simultaneous transmission to multiple users on the same subcarrier allows efficient exploitation of system resources and consequently higher system capacity. However, two significant issues should be considered during this kind of transmission:(1) how to minimize interference encountered by users on downlink (DL) transmission due to subcarrier sharing and (2) how to choose users that will be assigned on the same subcarrier especially when number of users is higher than number of transmitting antennas.

This combined problem (i.e. minimizing interference and user selection) has been widely investigated in the literature.
Many solutions have been proposed to minimize interference encountered by users due to subcarrier sharing such as Dirty Paper Coding (DPC) [4], Block Diagonalization (BD) [5] and beamforming $(\mathrm{BF})$. Zero Forcing beamforming (ZFBF) [6] has been widely accepted as a suboptimal solution for solving such complex allocation problems. The user selection problem based on ZFBF has been investigated in [7], [8] to maximize system capacity without any further constraints. The problem has been rather investigated considering fairness in [9]-[12] and considering quality of service (QoS) in [13].

In this paper, we propose a reduced complexity resource allocation algorithm for the downlink of MISO-OFDMA system that considers sum rate, minimum rate, QoS and fairness among users. QoS improvement through a minimum user rate allows better network access. Unlike [7]-[13], the proposed algorithm achieves efficient resource allocation among users with low execution time needed by this algorithm. The proposed algorithm also takes QoS into account by considering the minimum user rate requirement for each user. The proposed algorithm does not need subcarrier reallocation to fulfill QoS requirement which highly increases complexity as in [13]. The fairness among users is implicitly enhanced by considering minimum rate for each user. Although the proposed algorithm does not exaggerate in fairness preservation as in [9], [10], it provides an acceptable level of fairness compared to fairnessbased algorithm in [9]. In terms of system capacity, the proposed algorithm is expected to enhance both average sum rate and average minimum rate compared to the three reference algorithms discussed in this paper, i.e., the round robin (RR) and the existing ones in [9], [13].

The remaining part of the paper is divided as follows. System model and problem formulation are explained in Section II and III respectively. Proposed algorithm is described in Section IV. Simulation and results are provided in Section V. Finally the paper is concluded in Section VI.

\section{SySTEM MODEL}

We consider a single-cell system with one base station (BS) at the center of the cell equipped with $T$ transmitting antennas. System bandwidth $B$ is divided equally into $N_{c}$ subcarriers and $K$ users are uniformly distributed within the cell such that $K \geq T$. The complex channel gain vector between the BS and any user $k$ on subcarrier $n$ is defined as the $1 \times T$ 
vector $\mathbf{h}_{k, n}=\left[h_{k, n}^{1} h_{k, n}^{2} \cdots h_{k, n}^{T}\right]$. System model can then be formulated as

$$
\mathbf{y}_{n}=\mathbf{H}_{n} \mathbf{x}_{n}+\mathbf{z}_{n}
$$

where $\mathbf{H}_{n}=\left[\mathbf{h}_{1, n}^{t} \mathbf{h}_{2, n}^{t} \cdots \mathbf{h}_{K, n}^{t}\right]^{t}$ is the $K \times T$ complex channel gain matrix on subcarrier $n, \mathbf{x}_{n}=\left[x_{1, n} x_{2, n} \ldots x_{T, n}\right]^{t}$ is the $T \times 1$ transmitted vector on subcarrier $n, \mathbf{z}_{n}=$ $\left[z_{1, n} z_{2, n} \cdots z_{K, n}\right]^{t}$ is the $K \times 1$ circular symmetric complex Gaussian noise vector with zero mean and covariance matrix $\sigma_{n}^{2} \mathbf{I}_{K}$ and $\mathbf{y}_{n}=\left[y_{1, n} y_{2, n} \cdots y_{K, n}\right]^{t}$ is the $K \times 1$ received vector whose components are the received signals by different users $1,2, \cdots K$ respectively.

To allow simultaneous transmission to multiple users on the same subcarrier $n$, careful transmit beamforming is exploited at the transmitter such that the new system model is formulated as

$$
\mathbf{y}_{n}=\mathbf{H}_{n} \mathbf{W}_{n} \mathbf{D}_{n} \mathbf{s}_{n}+\mathbf{z}_{n}
$$

where $\mathbf{W}_{n}=\left[\mathbf{w}_{1, n} \mathbf{w}_{2, n} \cdots \mathbf{w}_{K, n}\right]$ is the $T \times K$ transmit beamforming matrix whose each component $\mathbf{w}_{k, n}=$ $\left[w_{k, n}^{1} w_{k, n}^{2} \cdots w_{k, n}^{T}\right]^{t}$ is the $T \times 1$ corresponding beamforming vector associated with user $k$ on subcarrier $n, \mathbf{D}_{n}$ is the $K \times K$ diagonal power allocation matrix and $p_{k, n}$ is the power allocated to user $k$ on subcarrier $n$ such that

$$
\mathbf{D}_{n}=\left(\begin{array}{cccc}
\sqrt{p_{1, n}} & 0 & \cdots & 0 \\
0 & \sqrt{p_{2, n}} & \cdots & 0 \\
\vdots & \vdots & \ddots & \vdots \\
0 & 0 & \cdots & \sqrt{p_{K, n}}
\end{array}\right)
$$

and $\mathbf{s}_{n}=\left[s_{1, n} s_{2, n} \cdots s_{K, n}\right]^{t}$ is the $K \times 1$ vector representing transmitted symbols to different $\mathrm{K}$ users. The received signal by user $k$ on subcarrier $n$ is then given by

$$
y_{k, n}=\mathbf{h}_{k, n} \mathbf{w}_{k, n} \sqrt{p_{k, n}} s_{k, n}+\sum_{\substack{j=1 \\ j \neq k}}^{K} \mathbf{h}_{k, n} \mathbf{w}_{j, n} \sqrt{p_{j, n}} s_{j, n}+z_{k, n}
$$

where the second term represents the interference encountered by different users due to simultaneous transmission on the same subcarrier $n$ and can be eliminated by smart beamforming as will be explained in next section.

\section{PROBLEM FORMULATION}

We assume that perfect channel state information (CSI) is available at the transmitter. Since the number of transmitting antennas $T \leq K$, so we need to choose $l \leq T$ users among the $K$ users on each subcarrier. Consequently, $I=\sum_{l=1}^{T}\left(\begin{array}{c}K \\ l\end{array}\right)$ possible configurations are available. Each of them is denoted as $A_{i}$ where $A_{i} \subset\{1,2, \cdots K\}$ and $0<\left|A_{i}\right| \leq T$ where $\left|A_{i}\right|$ is the cardinality of set $A_{i}$.

Assuming that the group of users $A_{i}$ are assigned to subcarrier $n$, then the received signal by any user $\left(k \in A_{i}\right)$ is given by

$y_{k, n}=\mathbf{h}_{k, n} \mathbf{w}_{k, n} \sqrt{p_{k, n}} s_{k, n}+\sum_{\substack{j \in A_{i} \\ j \neq k}} \mathbf{h}_{k, n} \mathbf{w}_{j, n} \sqrt{p_{j, n}} s_{j, n}+z_{k, n}$

where the second term represents the interference caused by users assigned on the same subcarrier $n$. Our proposed suboptimal strategy to eliminate such kind of interference is to use
Zero Forcing beamforming (ZFBF) at the transmitter such that the beamforming matrix $\tilde{\mathbf{W}}_{n}=\left[\tilde{\mathbf{w}}_{1, n} \tilde{\mathbf{w}}_{2, n} \cdots \tilde{\mathbf{w}}_{T, n}\right]$; whose columns are the beamforming precoding vectors associated with each user $\left(k \in A_{i}\right)$ on subcarrier $n$; is given by

$$
\tilde{\mathbf{W}}_{n}=\mathbf{H}_{n}\left(A_{i}\right)^{H}\left(\mathbf{H}_{n}\left(A_{i}\right) \mathbf{H}_{n}\left(A_{i}\right)^{H}\right)^{-1}
$$

where $\mathbf{H}_{n}\left(A_{i}\right)=\left[\mathbf{h}_{1, n}^{t} \mathbf{h}_{2, n}^{t} \cdots \mathbf{h}_{T, n}^{t}\right]^{t}$ is the channel matrix of users group $A_{i}$ on subcarrier $n$. For normalized power, each precoding vector is normalized such that

$$
\mathbf{w}_{k, n}=\sqrt{\frac{\left|A_{i}\right|}{\operatorname{tr}\left(\tilde{\mathbf{W}}_{n} \tilde{\mathbf{W}}_{n}^{H}\right)}} \tilde{\mathbf{w}}_{k, n}
$$

By using ZFBF, the term $\mathbf{h}_{k, n} \mathbf{w}_{j, n}=0$ for any $(j \in$ $\left.A_{i}, j \neq k\right)$ and hence the received signal by user $k$ on subcarrier $n$ is reduced to

$$
y_{k, n}=\mathbf{h}_{k, n} \mathbf{w}_{k, n} \sqrt{p_{k, n}} s_{k, n}+z_{k, n}
$$

By using M-QAM modulation, the data rate achieved by any user $k$ on subcarrier $n$ is given by

$$
r_{k, n}=\log _{2}\left(1+p_{k, n} \gamma_{k, n}\right)
$$

where $\gamma_{k, n}=\frac{\left|\mathbf{h}_{k, n} \mathbf{w}_{k, n}\right|^{2}}{\sigma_{n}^{2} \Gamma}, \Gamma=-\frac{\ln \left(5 B E R_{k}\right)}{1.5}$ [14] and $B E R_{k}$ is the target bit error rate for user $k$. The total achieved rate by user $k$ is then given by

$$
R_{k}=\sum_{n=1}^{N_{c}} \sum_{i=1}^{I} \rho_{k, n, i} \log _{2}\left(1+p_{k, n, i} \gamma_{k, n}\right)
$$

where $\rho_{k, n, i} \in\{0,1\}$ denotes whether subcarrier $n$ is assigned to user $k \in A_{i}(=1)$ or not $(=0)$. The optimization problem can then be formulated as

$$
\begin{array}{ll}
\underset{\rho_{k, n, i}, p_{k, n, i}}{\operatorname{maximize}} & \sum_{k=1}^{K} R_{k} \\
\text { subject to } & \rho_{k, n, i} \in\{0,1\} \quad \forall k, i, n \\
& p_{k, n, i} \geq 0 \quad \forall k, i, n \\
& \sum_{k=1}^{K} p_{k, n, i} \leq \frac{P_{\text {tot }}}{N_{c}} \quad \forall n, i \\
& \sum_{k=1}^{K} \rho_{k, n, i} \leq T \quad \forall n, i \\
& R_{k} \geq m r_{k} \quad \forall k
\end{array}
$$

where $P_{\text {tot }}$ is the total power available and $m r_{k}$ is the minimum target rate for any user $k$. This problem is an NPhard optimization problem with complexity of the order $I^{N_{c}}$, so we need to search for a sub-optimal solution for the problem based on ZFBF instead of optimal exhaustive search among all possible solutions.

\section{Proposed Resource Allocation Algorithm}

We proposed a reduced complexity suboptimal solution for the optimization problem mentioned in Section III based on ZFBF. The algorithm in details is described in Algorithm 1.

The proposed algorithm is an enhanced version of the algorithm in [9]. The algorithm is initialized with zero values 


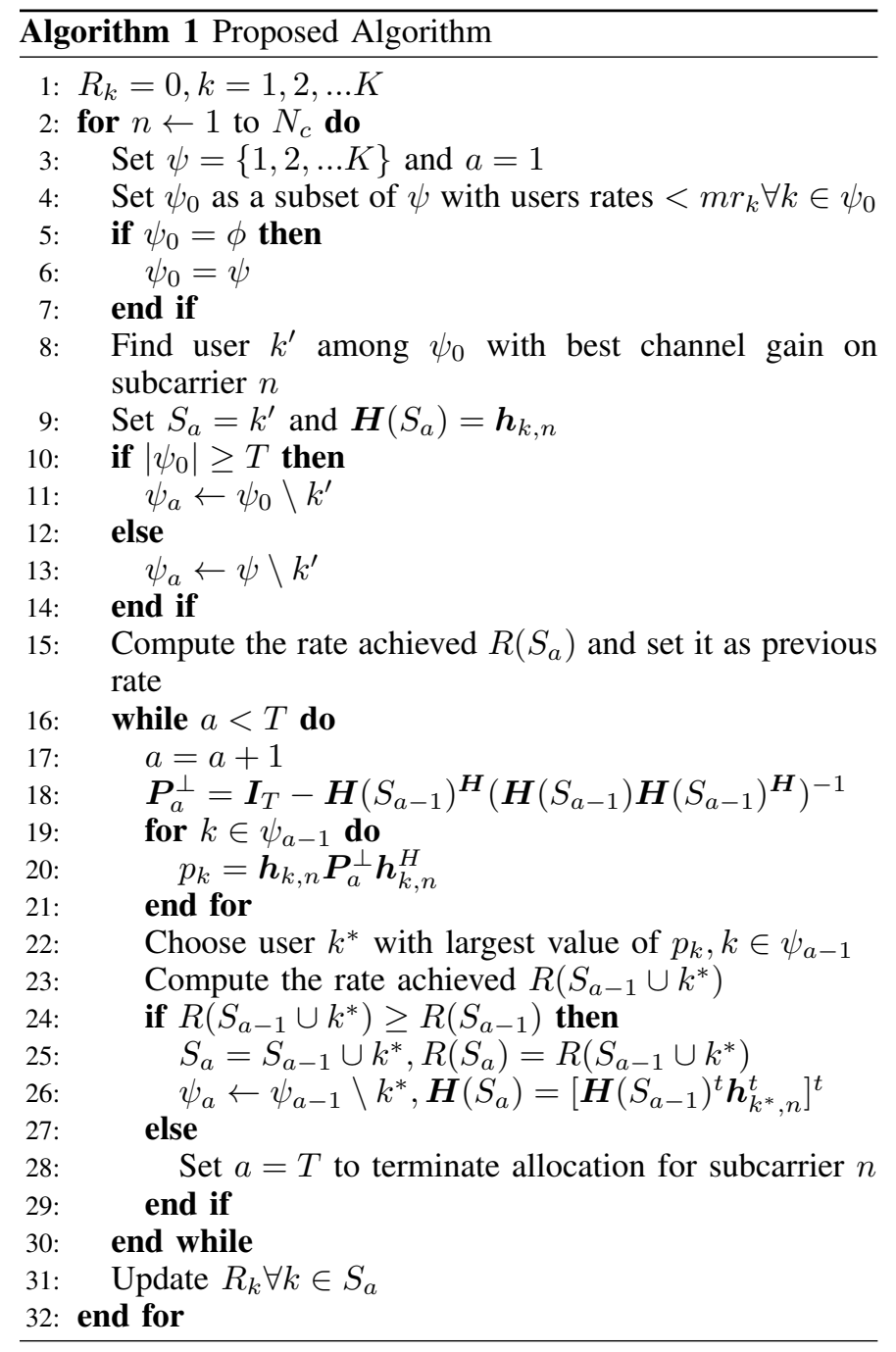

for user rates $R_{k}$ and the allocation process is done per subcarrier. At each iteration, users pool $\psi_{0}$ is defined as the set of users still with rates lower than minimum target rate $m r_{k}$. If all users are above rate, the pool $\psi_{0}$ becomes all users attached to BS. The parameter $a$ is initialized with 1 and used as a counter to count for number of users selected on each subcarrier during user selection step. Once no further gain is obtained on the cumulative rate of users assigned on any subcarrier, the parameter $a$ is set to $T$ to terminate the user selection loop and go to the rate update step. User $k^{\prime}$ with best channel conditions on subcarrier $n$ is chosen among pool of users to be assigned on subcarrier $n$. The remaining users of the pool (excluding $k^{\prime}$ ) $\psi_{a}$ are considered possible candidates for assignment on the same subcarrier $n$. To avoid lack of users and consequently rate decrease, let the set of users of possible candidates $\psi_{a}$ be the set of all users (excluding $k^{\prime}$ ) if the number of users in set $\psi_{0}$ becomes less than number of transmitting antennas. The rate achieved by user $k^{\prime}$ alone on subcarrier $n$ is calculated based on Equation (8) and the process of assigning more users with maximum $T$ users on each subcarrier $n$ begins.

We then calculate the projection matrix $\boldsymbol{P}^{\perp}$ to get the projection of channel vector of each candidate user belonging to $\psi_{a-1}$ on the orthogonal complement of subspace spanned by selected user(s) so far. The user $k^{*}$ among all possible users $\psi_{a-1}$ that maximizes the term $p_{k}$ is chosen as a possible candidate for allocation on subcarrier $n$. If the sum rate of selected users so far on subcarrier $n$ is increasing, user $k^{*}$ is admitted on subcarrier $n$. Then the process is repeated to allocate more users on subcarrier $n$ until maximum number $T$ is reached or the sum rate is no longer increasing. We then update the rates of all users selected on subcarrier $n$ based on Equations (8), (9) and the algorithm continues for another subcarrier until all subcarriers are allocated.

\section{Simulation ANd Results}

We simulate an MISO-OFDMA system with one BS of radius $1 \mathrm{Km}$ equipped by 4 transmitting antennas. Total transmit power $P_{t o t}$ is $43 \mathrm{dBm}$ and system bandwidth is divided into 64 subcarriers. Rayleigh fading channel model with 6 taps and power decay profile (PDP) with exponential decay is assumed and M-QAM is used as modulation type. The target $\mathrm{BER}_{k}$ is set to $10^{-3}$ and minimum user rate $m r_{k}=1.5 \mathrm{bit} / \mathrm{sec} / \mathrm{Hz}$. Number of channel realizations is 200 . We evaluate system performance in terms of sum rate, fairness, outage probability and finally the execution time for different algorithms as a measure of the required complexity.

We compare our proposed algorithm with two reference algorithms in [9] (referred in results as Alg [1]) and [13] (referred in results as Alg [2]), and with the round robin (RR) algorithm. The algorithm in [9] is a fairness based allocation algorithm that always starts with user with minimum rate so far. The user selection step is done among users that maximizes proportional fairness and provides lowest interference when assigned on the same subcarrier with that user. This algorithm highly exaggerates in fairness preservation at the expense of efficient resource allocation. The algorithm in [13] only considers system capacity during its first allocation step. After that, QoS is considered through minimum user rate for each user by a second reallocation step which is performed for users that fail to reach minimum rate during the first allocation. This algorithm requires significant increase in complexity to fulfil QoS requirement. In RR algorithm, each user is allowed for a fair amount of resources regardless of its channel conditions.

Figures 1 and 2 show the average sum rate of users per subcarrier and average minimum user rate per subcarrier against number of users respectively at $S N R=20 d B$. In Fig. 1, the proposed algorithm shows the best performance in terms of average sum rate among all techniques and even outperforms the greedy algorithm in [13] as it does not exaggerate in fairness guarantee as algorithm in [9] or waste resources as RR technique. In Fig. 2, the proposed algorithm shows a good performance in terms of average minimum user rate (measurement of both QoS and fairness) with slight drop below algorithm in [9] and outperforms all other techniques.

Figures 3 and 4 show the average users sum rate and minimum rate per subcarrier respectively for ( $K=10$ users) against SNR that varies from $5 \mathrm{~dB}$ to $40 \mathrm{~dB}$ with $5 \mathrm{~dB}$ step. The proposed algorithm shows the best performance in terms of average sum rate among the compared techniques as shown in Fig. 3 and shows a good performance (just below reference algorithm in [9]) in terms of minimum user rate for a wide range of SNR values up to $20 \mathrm{~dB}$ as shown in Fig. 4 while RR can outperform it at high SNR values. 


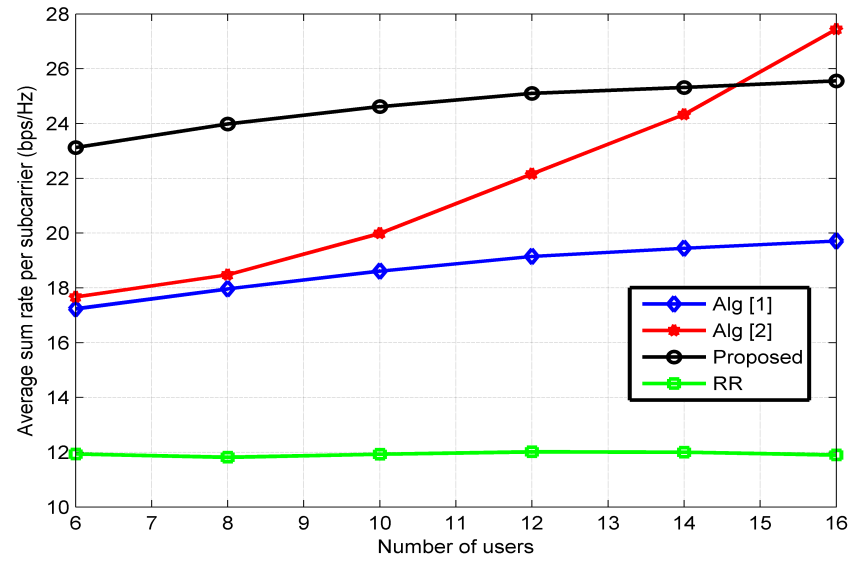

Fig. 1: Average sum rate vs. number of users $(\mathrm{SNR}=20 \mathrm{~dB}$ )

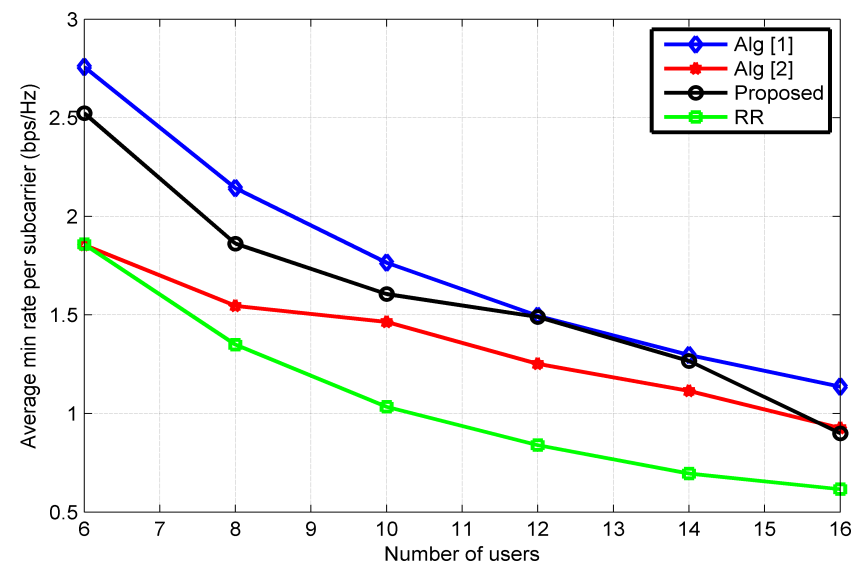

Fig. 2: Average minimum rate vs. number of users $(\mathrm{SNR}=20$ $\mathrm{dB})$

We compare the different algorithms in terms of fairness. Fig. 5 shows fairness index (FI) defined as $\mathbf{F I}=\frac{\left(\sum_{\mathrm{k}=1}^{\mathrm{K}} \mathbf{R}_{\mathbf{k}}\right)^{2}}{\mathbf{K} \sum_{\mathrm{k}=1}^{\mathrm{K}} \mathbf{R}_{\mathrm{k}}^{2}}$ [15] as a function of number of users at $S N R=20 \mathrm{~dB}$. Although proposed algorithm has the lowest performance among different techniques, it still provides good values of FI above 0.93 which is considered an acceptable level of fairness.

We also compare QoS of different algorithms through outage probability. Outage probability is defined as the percentage of users failed to fulfill the minimum target rate requirement $m r_{k}$. Figures 6 and 7 show outage probability against number of users (at $S N R=20 \mathrm{~dB}$ ) and SNR (with $K=10$ ) respectively. The proposed algorithm and reference algorithm in [13] consider minimum user rate during allocation process in contrast with the other two algorithms. Our proposed algorithm shows the best performance in terms of outage probability for any number of users as shown in Fig. 6 and all values of SNR as shown in Fig. 7 as the subcarriers are allocated efficiently without neither aggrieving users with poor channel conditions nor wasting resources on exaggerated fairness as in the case of RR and reference algorithm in [9].

We finally compare the complexity of different algorithms

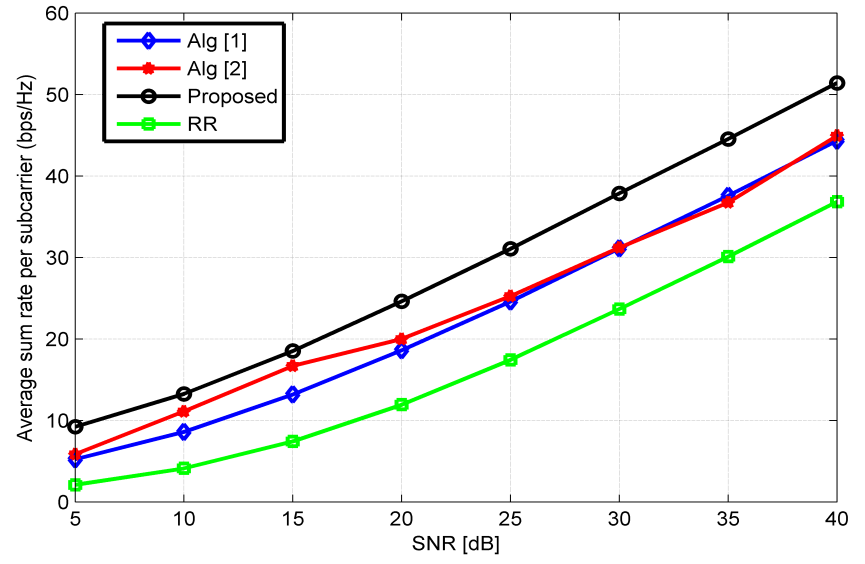

Fig. 3: Average sum rate vs. SNR $(\mathrm{K}=10)$

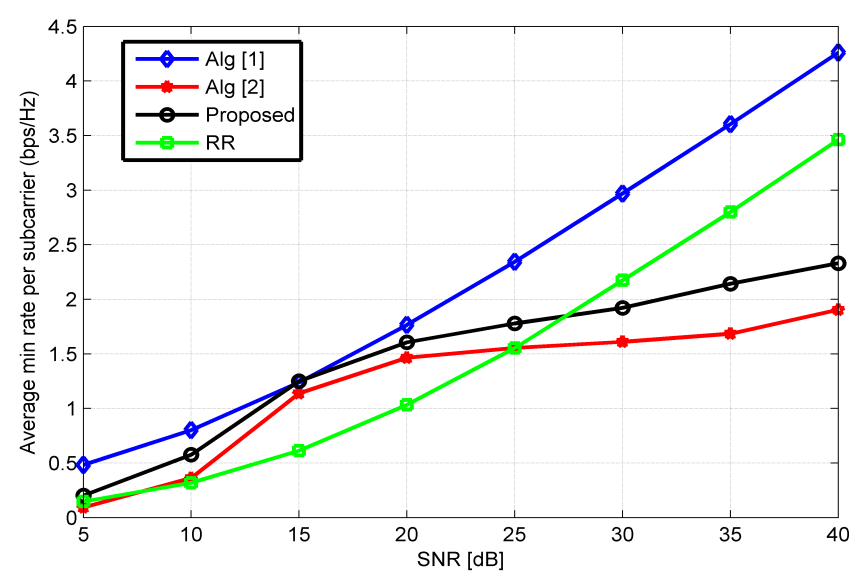

Fig. 4: Average minimum rate vs. SNR $(\mathrm{K}=10)$

through measuring the execution time needed by each of them. Table I represents the average execution time needed by each algorithm against number of users at $S N R=20 \mathrm{~dB}$. Results in Table I reveals that our proposed algorithm has lower complexity (measured through execution time) compared to the very complex algorithm in [13]. The proposed algorithm almost halved the execution time needed compared to reference algorithm in [9] and provided better performance based on performance metrics mentioned above. Intuitively, RR has the lowest complexity as it does not require any intelligence during allocation process as users are queued and allocated equally.

TABLE I: Average execution time (msec) under different number of users $(S N R=20 \mathrm{~dB})$

\begin{tabular}{c|c|c|c|c}
\hline Number of users & Algorithm in [9] & Algorithm in [13] & Proposed & RR \\
\hline \hline $\mathrm{K}=6$ & 157.4 & 185.7 & 101.2 & 9.5 \\
\hline $\mathrm{K}=8$ & 176.1 & 503.5 & 110.3 & 9.3 \\
\hline $\mathrm{K}=10$ & 187.2 & 1289.9 & 118.4 & 9.3 \\
\hline $\mathrm{K}=12$ & 197.5 & 2517.8 & 125.3 & 9.3 \\
\hline $\mathrm{K}=14$ & 208 & 3744 & 131.1 & 9.3 \\
\hline $\mathrm{K}=16$ & 218.5 & 5425.9 & 136.6 & 9.3 \\
\hline \multicolumn{4}{|r}{}
\end{tabular}




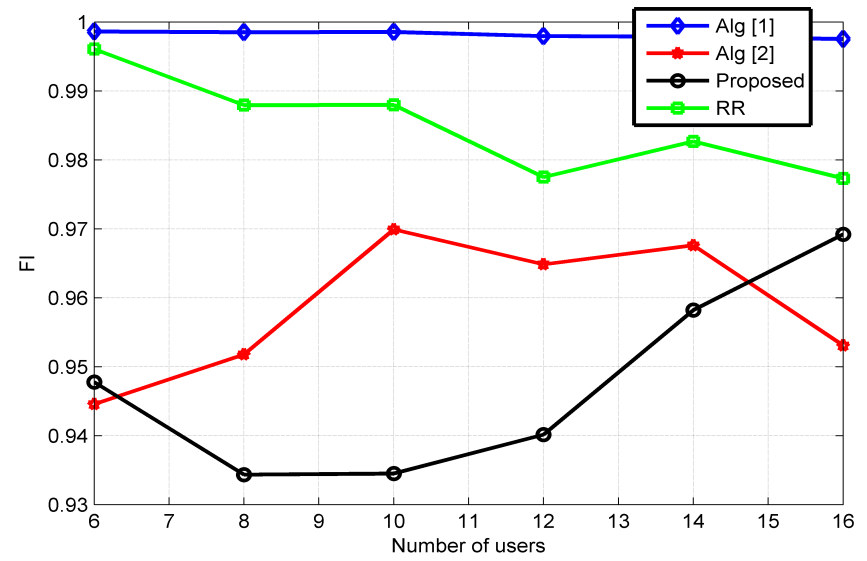

Fig. 5: Fairness index vs. number of users $(\mathrm{SNR}=20 \mathrm{~dB})$

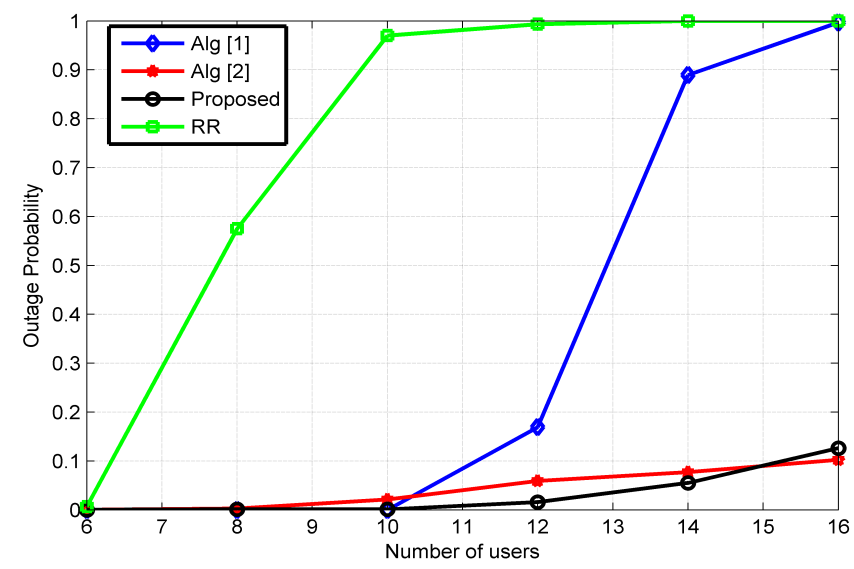

Fig. 6: Outage probability vs. number of users $(\mathrm{SNR}=20 \mathrm{~dB})$

\section{CONCLUSION}

We proposed a resource allocation algorithm for the downlink of MISO-OFDMA system based on ZFBF. The proposed algorithm has better performance compared to reference algorithms in terms of average users sum rate. Our proposed algorithm also considers QoS (measured by outage probability) by preserving minimum user rate for each user that allows better network access and higher data services for users. The algorithm has a good performance for wide range of SNR values without degrading fairness among users. The complexity of proposed algorithm has been measured through execution time needed and proved to have reduced complexity compared to reference algorithms except the round robin algorithm which is the simplest but has the worst QoS.

\section{ACKNOWLEDGMENT}

The authors would like to thank the ministry of higher education (MoHE)-missions department, and Egypt-Japan University of Science and Technology (E-JUST) for funding our work.

\section{REFERENCES}

[1] S. Pietrzyk, OFDMA for broadband wireless access. Artech House Boston, 2006.

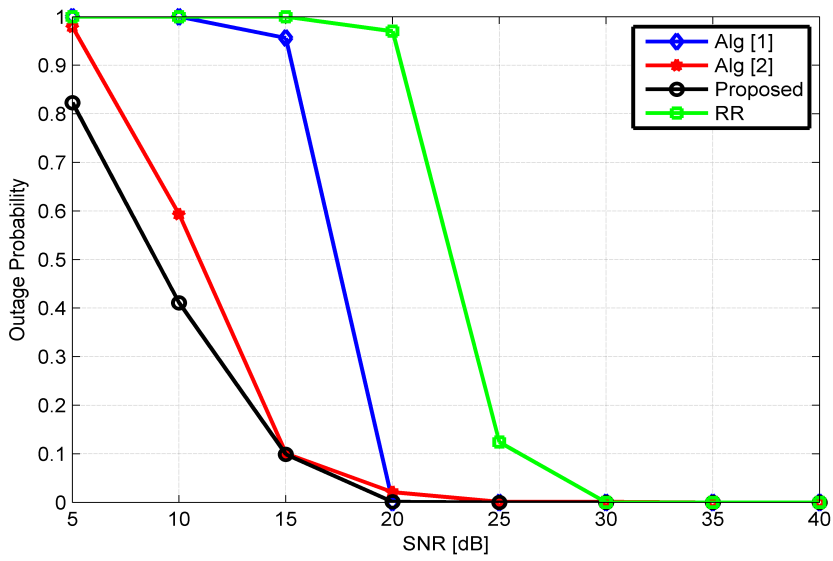

Fig. 7: Outage probability vs. SNR $(\mathrm{K}=10)$

[2] H. Weingarten, Y. Steinberg, and S. Shamai, "The capacity region of the Gaussian MIMO broadcast channel," in Information Theory, 2004. ISIT 2004. Proceedings. International Symposium on. IEEE, 2004, p. 174.

[3] D. L. Ruyet and B. Ozbek, "Resource Management Techniques for OFDMA," in Orthogonal Frequency Division Multiple Access Fundamentals and Applications. Auerbach Publications, 2010.

[4] M. Costa, "Writing on dirty paper (corresp.)," IEEE Transactions on Information Theory, vol. 29, no. 3, pp. 439-441, 1983.

[5] Z. Shen, R. Chen, J. G. Andrews, R. W. Heath, and B. L. Evans, "Low complexity user selection algorithms for multiuser MIMO systems with block diagonalization," IEEE Transactions on Signal Processing, vol. 54, no. 9, pp. 3658-3663, 2006.

[6] Q. H. Spencer, A. L. Swindlehurst, and M. Haardt, "Zero-forcing methods for downlink spatial multiplexing in multiuser MIMO channels," IEEE Transactions on Signal Processing, vol. 52, no. 2, pp. 461-471, 2004.

[7] M. Petermann, C. Bockelmann, and K.-D. Kammeyer, "On allocation strategies for dynamic MIMO-OFDMA with multi-user beamforming," in Proceedings of 12th International OFDM-Workshop-InOWo07, 2007.

[8] Z. C.-x. Y. Lu-xi, "Dynamic resource allocation for downlink multiuser MIMO-OFDMA/SDMA systems [j]," Journal of Electronics \& Information Technology, vol. 12, p. 042, 2008.

[9] I. Basturk and B. Ozbek, "Fairness aware resource allocation for downlink MISO-OFDMA systems," in Wireless Communications and Networking Conference (WCNC), 2012 IEEE, 2012, pp. 188-192.

[10] K. Sun, Y. Wang, Z.-X. Chen, and P. Zhang, "Fairness based resource allocation for multiuser MISO-OFDMA systems with beamforming," The Journal of China Universities of Posts and Telecommunications, vol. 16, no. 1, pp. 38-43, 2009.

[11] V. D. Papoutsis, I. G. Fraimis, and S. A. Kotsopoulos, "User selection and resource allocation algorithm with fairness in MISO-OFDMA," IEEE Communications Letters, vol. 14, no. 5, pp. 411-413, 2010.

[12] V. D. Papoutsis and S. A. Kotsopoulos, "Chunk-based resource allocation in distributed MISO-OFDMA systems with fairness guarantee," IEEE Communications Letters, vol. 15, no. 4, pp. 377-379, 2011.

[13] V. Papoutsis and S. Kotsopoulos, "Resource allocation algorithm for MISO-OFDMA systems with QoS provisioning," in ICWMC 2011, The Seventh International Conference on Wireless and Mobile Communications, 2011, pp. 7-11.

[14] A. J. Goldsmith and S.-G. Chua, "Variable-rate variable-power M-QAM for fading channels," IEEE Transactions on Communications, vol. 45, no. 10, pp. 1218-1230, 1997.

[15] R. Jain, D.-M. Chiu, and W. R. Hawe, A quantitative measure of fairness and discrimination for resource allocation in shared computer system. Eastern Research Laboratory, Digital Equipment Corporation, 1984. 